\title{
Legray, Bodson, Varlet. Amitié politique et relations privées. Deux documents inédits
}

Jean-Jacques Tomasso

\section{(2) OpenEdition \\ 1 Journals}

\section{Édition électronique}

URL : https://journals.openedition.org/ahrf/13187

DOI : $10.4000 /$ ahrf. 13187

ISSN : 1952-403X

Éditeur :

Armand Colin, Société des études robespierristes

\section{Édition imprimée}

Date de publication : 1 juin 2014

Pagination : 179-190

ISBN : 978-2-908327-68-7

ISSN : 0003-4436

Référence électronique

Jean-Jacques Tomasso, « Legray, Bodson, Varlet. Amitié politique et relations privées. Deux documents inédits », Annales historiques de la Révolution française [En ligne], 376 | avril-juin 2014, mis en ligne le 01 juin 2017, consulté le 01 juillet 2021. URL : http://journals.openedition.org/ahrf/13187 ; DOl : https://doi.org/10.4000/ahrf.13187 


\title{
LEGRAY, BODSON, VARLET. AMITIÉ POLITIQUE ET RELATIONS PRIVÉES DEUX DOCUMENTS INÉDITS
}

\author{
Jean-Jacques TOMASSO
}

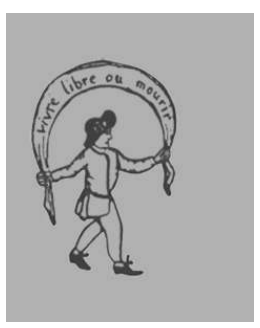

La destinée des cadres politiques du mouvement révolutionnaire parisien, tout au moins pour ceux qui échappèrent aux exécutions et aux vagues de déportations, est très mal connue. Quelle fut leur vie ? Continuèrent-ils à se fréquenter, à s'entraider ? Les amitiés politiques qui s'étaient nouées durant la Révolution se muèrent-elles, dans l'adversité, en solidarités ?

Le peu que l'on en sache provient essentiellement de sources policières ou judiciaires (rapports d'espions, pièces de procès, dossiers de déportation, etc.); témoignages toujours suspects quant aux relations que les anciens révolutionnaires auraient maintenues entre eux, tant les policiers étaient prompts à inventer des complots ou des conciliabules afin de justifier une répression strictement politique. Il est de fait extrêmement rare de retrouver des documents de la sphère privée attestant de manière irréfutable des liens personnels ayant existé entre certains révolutionnaires, permettant de mieux cerner la cohésion de ces groupes, de ces réseaux informels, dont on devine le rôle important lors des grands événements de la Révolution. Ainsi au 9 Thermidor où, la suspicion politique ayant atteint son paroxysme, l'attitude déterminante des dirigeants sectionnaires fut, dans l'urgence, davantage dictée par les relations de confiance ou de défiance qu'ils avaient entre eux, que par une analyse politique et une vision claire de l'issue de la crise.

Militants révolutionnaires depuis 1789, François Vincent Legray, Joseph Bodson et Jean François Varlet avaient été de toutes les grandes journées parisiennes. Membres des Cordeliers, élus par leur section respective à la Commune du 10 août puis à l'assemblée électorale de Paris... Durant toute la Révolution, ils ne renièrent pas leurs convictions, ni après la 
chute d'Hébert et de Chaumette ni après leur séjour en prison en l'an II. Ils continuèrent à lutter avec ténacité contre l'instauration du gouvernement révolutionnaire et pour la mise en œuvre de la constitution de 1793, allant jusqu'à soutenir activement les thermidoriens qui les renvoyèrent en prison dès qu'ils ne leur furent plus utiles. S'ils sont souvent cités ensemble, c'est qu'ils incarnent la continuité du mouvement sans-culotte de 1789 à 1795, devenant tous trois, avec Babeuf, les principaux dirigeants du Club électoral qui reprit momentanément, après le 9 Thermidor, le rôle des Cordeliers face au moribond Club des jacobins ; puis, devenant d'actifs organisateurs du soulèvement de Prairial, révolte de la faim à laquelle ils joignirent leurs revendications politiques et qui restera résumée dans le célèbre mot d'ordre « Du pain et la Constitution de 1793 ».

Ces « thermidoriens de gauche », ces « néo-hebertistes » ont pu être considérés, faute de documents probants, comme un groupe hétéroclite dont l'unité n'était que de circonstance, dictée avant tout par les vagues de répression qu'ils subirent plusieurs fois en commun. Mais de réels liens d'amitié et de confiance s'étaient tissés entre eux, comme le révèlent quelques documents inédits, mis au jour lors de recherches pour tenter de reconstituer leurs biographies ${ }^{1}$.

La première pièce, conservée au Minutier central des notaires parisiens aux Archives nationales, datée du $1^{\mathrm{er}}$ jour complémentaire an $\mathrm{V}$ (14 septembre 1797), est le contrat de mariage de Legray qui épousait le lendemain la sœur cadette des frères Bodson, Angélique Thérèse ${ }^{2}$. Mais

(1) Seul Varlet a fait l'objet de quelques recherches, mais sa biographie est encore bien lacunaire. Voir Morris SLAVIN, «L'autre Enragé : Jean-François Varlet », Eine Jury für Jacques Roux. Dem Wirken Walter Markovs gewidmet, Sitzungsbericht der Akademie der Wissenschaften der DDR, Berlin, 1981, p. 34-67 ; J.-M. ZACKER, « Jean Varlet pendant la réaction thermidorienne... », AHRF, 1961, p. 19-34 ; Yves BLAVIER, « Jean-François Varlet après la Révolution », AHRF, 1991, p. 227-231. J'espère pouvoir bientôt compléter ces travaux grâce à de nombreuses pièces d'archives inédites. Quant à Legray, seule l'affaire de son incarcération au début de thermidor an II a été étudiée. D'abord par Mathiez qui utilisait le dossier de police de Legray ( $\mathrm{F}^{7} 4774$ 14) dans « La campagne contre le gouvernement révolutionnaire à la veille du 9 Thermidor : L'affaire Legray », AHRF, 1927, p. 309-325 ; puis, par Pierre Caron qui complétait l'article de Mathiez à l'aide du dossier de Legray au Tribunal révolutionnaire (W 47, d. 3138) dans «L'opposition de gauche à la veille du 9 thermidor », $A H R F$, 1947 , p. 322-325.

(2) Les Bodson étaient une grande fratrie, deux filles et six garçons, enfants de Henry Bodson, orfèvre bijoutier, et de Jeanne Antoinette Duhamel, polisseuse de bijoux. Ils perpétuèrent la tradition familiale devenant tous artisans d'art, joailliers, opticiens, bijoutiers ou graveurs. Plusieurs furent d'actifs militants dans leur section. La liste de cette fratrie nous est donnée, en l'an V, par un acte de notoriété des héritiers de l'un d'eux, Charles Henry (fondeur, alors installé à Angoulême), acte pour lequel Legray fut témoin (AN, ET LXVI 717). Le plus connu par son engagement révolutionnaire, Joseph Bodson, était né à Paris le 3 mars 1768, graveur (il réalisa plusieurs affiches pour Babeuf), peintre sur porcelaine, et peintre en décor. Il vécut à partir de 1821 au 29 rue de la Grange-aux-Belles, et décéda à la maison de santé de $\mathrm{M}^{\mathrm{me}}$ Bonnemain-Chértet, à La Glacière, commune de Gentilly, le 27 juin 1835. 
cette union fut de bien courte durée. Un mois plus tard, le 29 vendémiaire an VI (20 octobre 1797), Legray décédait à son domicile, au 1 de la rue des Deux-Boulles ${ }^{3}$.

La seconde pièce, conservée aux Archives départementales de Paris, est le procès-verbal du juge de paix, Jean Le Sèvre, qui consignait non seulement le décès de Legray mais aussi la liste des opposants "à la reconnaissance et levée des scellés autrement qu'en leur présence » afin de faire valoir leurs droits. Parmi eux, Jean François Varlet, alors retiré à la campagne, dont nous apprenons qu'il avait confié à Legray tous ses papiers et la gestion de ses biens.

\section{Contrat de mariage entre François Vincent Legray et Angélique Thérèse Bodson ${ }^{4}$}

« $1^{\mathrm{er}}$ jour complémentaire an $\mathrm{V}$

Par-devant les notaires soussignés furent présents François Vincent Legray dem ${ }^{t}$ à Paris rue des Deux Boules $n^{0} 1$, division du Museum, fils majeur de Antoine Charles Legray ${ }^{5}$ et de Charlotte Françoise Gousset son épouse à présent sa veuve, stipulant, le $\mathrm{C}^{\mathrm{n}}$ Legray, pour lui et en son nom de l'agrément de sa mère dem ${ }^{\text {te }}$ à Paris susdite rue des Deux-Boules $\mathrm{n}^{\mathrm{o}} 1^{\mathrm{er}}$, pour ce présente, d'une part

Et Angélique Thérèse Bodson dem ${ }^{\text {te }}$ à Paris, quai de l'Horloge du Palais $\mathrm{n}^{\mathrm{o}} 48^{6}$, division du Pont Neuf, fille majeure de Henry Bodson et de Jeanne Duhamel, son épouse, stipulant, la fille Bodson, pour elle et en son nom, d'autre part

Lesquels dans la vue du mariage qu'ils sont sur le point de contracter sont convenus d'en régler les effets civils de la manière suivante, en présence de leurs parens et amis cy après nommés, savoir du côté de la

(3) Ce mariage était sans doute la régularisation d'une vie commune antérieure ; régularisation que la maladie de Legray rendait sans doute nécessaire. Ils se connaissaient en tout cas depuis longtemps. Le 30 juillet 1790, deux tantes d'Angélique, Geneviève et Marie Marguerite Duhamel, s'étaient constitué une rente de 224 livres, sur un capital de 4494 livres, qui devait revenir à leur nièce après leur décès. C'est Legray qui régla l'opération : "François Vincent Legray, bourgeois de Paris y demeurant, place du Chevalier du Guet, paroisse Saint Germain l'Auxerrois, au présent acquéreur pour lesdites Dlles Duhamel et Bodson... » (AN, ET XIX 897).

(4) AN, ET LXVI 717, acte passé devant $\mathrm{M}^{\mathrm{e}}$ Louis André Clairet, notaire, rue des Bourdonnais, au coin de la rue des Mauvaises-Paroles.

(5) Antoine Charles Legray était marchand mercier. Après la faillite de son commerce et la saisie de ses biens en 1761, il avait entraîné sa famille dans la désastreuse aventure organisée par Choiseul d'aller peupler en masse la Guyane pour en faire une colonie prospère. Son épouse, Charlotte Françoise Gousset, en revint, veuve, avec seulement deux des quatre enfants qu'ils y avaient emmenés.

(6) C'était l'adresse familiale où vivaient encore sa mère, plusieurs de ses frères et une de ses tantes. 
future épouse, de Jean Joseph Bodson, dem ${ }^{t}$ à Paris susdit quai de l'Horloge du Palais $n^{0} 28$, frère de la future

De Marguerite Duhamel, fille majeure, dem $^{\text {te }}$ à Paris susdit quai de l'Horloge, sa tante maternelle

Et de Jeanne Thérèse Wibert, épouse de Edmé Bodson, belle sœur de la future épouse,

$[-]^{7}$

Les futurs époux seront communs de bien meubles et conquêts, immeubles suivant la coutume de Paris [...]

Les futurs époux se prennent avec les biens appartenant à chacun d'eux consistant, ceux du futur époux en une somme de six mille livres en meublans argenterie habits linge et hardes et deniers comptant provenant de ses gains et épargnes et dans lesquels sa dite mère n'a contribué en aucune manière, ainsi qu'elle le reconnaît ; et ceux de la future épouse, en une somme de quatre mille livres, en habit, linge, et hardes, bijoux, dentelles et deniers comptant provenant de ses gains et épargnes dont le futur époux sera chargé par le fait de son mariage.

Des biens des futurs époux, il entrera de part et d'autre en communauté, la somme de douze cent livres, ce qui fera deux mille quatre cent livres pour les deux, et quant au surplus il demeurera propre de chaque coté ainsi que ce qu'il leur adviendra pendant le mariage en meuble et immeubles par succession, donations, legs ou autrement.

Le futur époux doüe la future épouse de trois mille livres de douaire préfix une fois versée dont elle jouira dès qu'il aura lieu suivant la coutume de Paris sans être tenu d'en faire demande en justice ni de donner caution.

[suivent les clauses habituelles de la coutume de Paris]

Fait et passé à Paris en la demeure de la future épouse cy dessus désignée en l'an cinq de la république française une et indivisible, premier des jours complémentaires, et ont signé les présentes. »

\section{Procès-verbal après décès de François Vincent Legray ${ }^{8}$}

« L'an six de la République française une et indivisible, le vingt-neuf vendémiaire, quatre heures du soir en notre demeure et par-devant nous,

(7) Un espace avait été laissé pour nommer les témoins du futur époux, qui ne fut pas rempli.

(8) AD Paris, D4 U1 4, Justice de paix, section du Muséum (4e arrondissement ancien), 58 p. Nous n'en reproduisons que l'essentiel. 
Jean Le Sèvre ${ }^{9}$, juge de paix de la division du Muséum, assisté du citoyen Pierre Joseph Ango notre secrétaire greffier par intérim.

Est comparu la citoyenne Angélique Thérèse Bodson, femme du citoyen François Vincent Legray et commune en bien avec luy, demeurant à Paris rue des Deux-Boulles, $n^{\circ} 1$, de cette section.

Laquelle nous a dit que François Vincent Legray, son mari, receveur des rentes est décédé ce jourd'huy entre trois et quatre heures du soir, en conséquence, attendu qu'il a un frère son présomptif héritier absent ${ }^{10}$, nous a requis de nous transporter au domicile dudit défunt Le Gray sis rue des Deux Boulles $n^{0} 1$, à l'effet d'apposer nos scellés et cachets sur ses meubles et effets, et de faire la description des objets que nous laisserons en évidence, le tout à la conservation des droits de qui il appartiendra, et a signé sous toute réserve de droit.

[signé] A T Bodson f [emme] Legray

Desquelles comparution, dire, réquisitions et réserves, nous avons donné acte, en conséquence disons que nous allons à l'instant nous transporter au domicile dudit défunt Le Gray à l'effet d'apposer nos scellés, et cachets sur les meubles et effets, et de faire la description des objets que nous laisserons en évidence, le tout à la conservation des droits de qui il appartiendra, pourquoy nous avons signé avec notre secrétaire greffier,

[signé] Angot, Lesèvre

En exécution de notre ordonnance cy dessus, nous nous sommes transportés avec la ditte citoyenne Le Gray susditte rue des Deux-Boulles

(9) Le Sèvre, ancien membre du comité civil de la section du Muséum, et Legray, ancien commissaire révolutionnaire de la même section, se connaissaient bien pour avoir longtemps milité ensemble.

(10) Armand Charles Legray, né en 1751, était devenu comédien « attaché à l'entreprise du théâtre de Molière », puis, le $1^{\text {er }}$ décembre 1792, il avait été embauché dans la $6^{\mathrm{e}}$ division du département de la Guerre dirigée par Xavier Audoin, au bureau des nominations de l'infanterie, sans doute sur la recommandation de son frère ; il démissionna de ce poste en l'an IV. Lors du décès de François Vincent, il vivait à Dunkerque. Revenu à Paris, il recueillit sa mère dans son appartement, cul-de-sac Laurent à la porte Saint-Denis, où elle décéda peu de temps après, le 26 nivôse an VII (15 janvier 1799) (AN, ET LXVI 721). 
$\mathrm{n}^{\mathrm{o}} 1$, dans une maison dont la citoyenne veuve Hesmart ${ }^{11}$ est propriétaire, où étant dans une chambre au troisième étage ayant vue sur la rue des Lavandières, nous avons trouvé étendu sur un lit un cadavre masculin qu'on nous a dit être celuy de défunt François Vincent Legray, et après avoir reçu de laditte femme Le Gray et de Marie Marguerite La Montagne, garde malade, demeurante quay de l'École ${ }^{0} 11$, le serment qu'elles n'ont rien pris, caché ou détourné des effets dépendant de la succession dudit défunt sous les peines de droit que nous leur avons expliqué et qu'elles ont dit bien comprendre, nous avons procédé à l'apposition de nos scellés et cachets...

[vient ensuite la description minutieuse de l'apposition des cachets et rubans sur un bureau en bois de noyer à trois tiroirs, une armoire à deux battants, sur la porte du cabinet et sur la porte de l'entrée de la cave]

...déclare, la citoyenne Le Gray, que le secrétaire étant dans le cabinet appartient à son beau-frère ainsi que tous les effets qu'il contient, et a signé

Description des objets laissés en évidence dans le cabinet :

Un feu de cheminée, chenets, etc. ; deux fauteuils de paille, un fauteuil de cabinet, six chaises de paille, vingt estampes de différentes grandeurs dans leur bordure de bois doré, six chandeliers de cuivre argenté, deux corps de bibliothèque, une pendule, un bureau de bois de noyer garni de ses tiroirs... dix tablettes pleines de livres dans les deux corps de bibliothèque, deux tasses et un sucrier de faïence, [...]

Suit la description des objets laissés en évidence dans la chambre à coucher :

Une cheminée à la prussienne, pelle etc., pendule, quatre flambeaux, [etc] une commode de bois d'acajou, secrétaire, table de chevet à dessus en marbre, un lit complet composé d'un sommier de crin, un lit de plume, deux matelas couverts de toile à carreaux, deux couvertures de laine blanche, une courtepointe d'indienne et les rideaux pareils, quatre chaises de paille.

Déclare la citoyenne Le Gray que les quatre fauteuils de velours d'Utrech appartiennent à son beau-frère.

(11) C'était la mère d'un héros éphémère du 9 thermidor : Jean Hesmart, marchand de drap et chef de la $29^{\mathrm{e}}$ légion de gendarmerie (il habitait rue de la Monnoye et sa boutique était rue du Roule). Nommé commandant de la force armée de Paris dans la matinée du 9 Thermidor en remplacement de Hanriot, il fut arrêté par ce dernier dès 15 heures, les Comités le remplacèrent alors par Fauconnier, chef de la $1^{\text {re }}$ légion. Sur Hesmart voir l'ouvrage publié par Gratien JAY-DUFRENOY, Mélanges historiques. Un chapitre inédit du 9 thermidor..., Dentu, 1885 ; et l'étude minutieuse réalisée par Arthur Birembaut, « Hesmart et son rôle au 9 thermidor », $A H R F, 1959$, p. 306-327. Si Legray était locataire de Madame Hesmart, mère, le juge de paix Le Sèvre était quant à lui locataire du fils, principal locataire de la maison du 39 rue de la Monnoye (bail du 9 juin 1790, AN, ET LXVI 692). 
Suivent les objets laissés en évidence dans l'antichambre :

Un buffet, une toilette de bois de noyer garnie, une petite table, une table à manger de bois blanc, etc.

Suivent les objets laissés en évidence dans la salle à manger servant de cuisine :

Un chiffonnier, une grande armoire et une table pliante, ustensiles de cuisine, etc.

Les scellés ont été laissés à la garde de la citoyenne femme Le Gray et de Jean-Joseph Bodson, demeurant quay de l'Horloge $n^{\circ} 29 .$.

Nous avons clos le présent procès-verbal après avoir vaqué à ce que dessus jusqu'à dix heures sonnées et ce fait en présence de la citoyenne Charlotte Françoise Gousset, veuve du citoyen Le Gray père, demeurant même maison [...]

Suit la liste des personnes qui se déclarent « opposans et s'opposent par les présentes à la reconnaissance et levée des scellés autrement qu'en leur présence »]

... 6 brumaire an VI, Pierre René Lemoine, ouvrier imprimeur demeurant à Paris rue de la Heaunerie $\mathrm{n}^{0}$ 9, division des Lombards ;

... 7 brumaire, Thomas Digard, boulanger, 10 rue Saint Germain l'Auxerrois ;

Et le nonidi 9 brumaire an septième de la république une et indivisible, Jean François Varlet, cy devant employé à l'administration des postes, demeurant à Pailly, canton de Sergines, département de l'Yonne, de présent à Paris, rue Tiron, $\mathrm{n}^{\mathrm{o}} 6$ division des droits de l'Homme

... 10 brumaire, Nicolas Vial, rue Meslay, comme fondé de la procuration de Germain Charles Lautran Duval, demeurant au Havre ;

Enfin le 12 brumaire, Angélique Thérèse Bodson, veuve de François Vincent Le Gray...

Laquelle nous a requis de nous transporter les jours et heures qu'il nous plaira indiquer au domicile du défunt Le Gray son mary à l'effet de procéder à la reconnaissance des scellés...

[ce qui est décidé pour le lendemain 13 brumaire]

Sont comparus :

$\left.1^{\circ}\right)$ Angélique Thérèse Bodson, veuve de Vincent François Le Gray, receveur des rentes demeurant à Paris dans la maison où nous sommes, en son nom et à cause de la communauté des biens qui a existé entre elle et son défunt mari au terme du contrat de mariage [...] laquelle communauté elle se réserve d'accepter ou de répudier par la suite ainsi qu'elle avisera, 
et encore comme donataire universelle en usufruit de son mari sans être assujetie de donner caution selon ledit contrat de mariage qui sera insinué incessamment ;

$2^{\circ}$ ) Félix Victor Dechevrières demeurant à Paris rue de la Harpe, $n^{\circ} 124$, division du Théâtre Français, au nom et comme fondé de la procuration spéciale à l'effet des présentes, d'Armand Charles Le Gray, artiste dramatique, demeurant à Dunkerque, ladite procuration passée devant Dusse et son confrère, notaires à Dunkerque le quatre frimaire, présent mois dont le brevet original duement enregistré est demeuré annexé à la minute dont il sera cy après parlé.

Ledit Charles Le Gray habile à se dire et porter unique héritier dudit François Vincent Le Gray, son frère germain.

$[\ldots]$

- est aussi comparu : René Le Moine, ouvrier imprimeur.... pour ce qui peut lui être dû par la succession du défunt Le Gray, pour le prix d'une inscription d'une rente perpétuelle de trois cents livres sur le grand livre [de la dette publique] qu'il avait chargé ledit Le Gray de vendre par la voye du transfert et que ce dernier luy a dit qu'il luy a dit avoir vendu avant son décès, moyennant le prix et somme de quinze cent francs numéraire, non compris les arrérages échus de la ditte rente, et en outre à l'effet de réclamer des titres, papiers et renseignements confiés audit défunt, relatifs à un arbitrage convenu entre le comparant et le citoyen Poiré son beau frère, lesdits papiers remis audit défunt en qualité d'arbitre nommé par le comparant...

- Est aussi comparu le citoyen Nicolas Vial [mandataire de Duval du Havre] à l'effet de réclamer quatre inscriptions sur le grand livre de la dette publique [une de 1000 livres, deux de 500 et une de 160]

- Est aussi comparu Marie Anne Catherine Joseph Duchemin, veuve d'Etienne Michel Bidault demeurant rue Eloy $n^{\circ} 19$ [pour] quatre inscriptions sur le grand livre de la dette publique de trois cents livres de rente chacune et d'autres...

[Procuration de Germain Charles Lautran Duval, Grande-Rue au Havre devant De Grau, notaire au Havre en faveur de Nicolas Vial, rue St Eloy à Paris]

- est aussy comparu Marie Anne Piat veuve de Bruno Clément, rue des Vieilles Etuves...

- est aussy comparu Jean François Varlet cy devant employé à l'administration des postes, demeurant à Pailly, canton de Sergine, département 
de $\mathrm{l}^{\prime}$ Yonne, de présent à Paris, logé rue Tiron $\mathrm{n}^{\mathrm{0}} 6^{12}$, division des Droits de l'Homme... lequel nous a dit que les causes de son opposition sont $\left.1^{\circ}\right)$ à l'effet de réclamer une inscription de douze cent cinquante livres de rente perpétuelle en son nom, qu'il a laissée audit défunt Le Gray pour être vendue, $2^{\circ}$ ) pour sûreté conservatoire et avoir payement en deniers ou quittances valables des loyers d'une maison sise à Paris rue des Fossés Saint Germain ${ }^{13}$ que ledit défunt Le Gray a été chargé de recevoir pour le comparant, $3^{\circ}$ ) à l'effet de réclamer un carton étiqueté en son nom contenant des titres, papiers et renseignements ${ }^{14}$ appartenants audit comparant, déclarant qu'il n'empêche qu'il ne soit procédé à la reconnaissance et levée des scellés...

- est aussy comparu Jean Joseph Bodson, demeurant à Paris quay de l'Horloge du Palais, $\mathrm{n}^{\mathrm{O}}$ 29, gardien de nos scellés conjointement avec lad[itte] veuve Le Gray...

Et le lendemain 14 brumaire...

- est comparu Marie Margueritte Bachelot veuve de Charles Nicolas David, cy devant tapissier demeurant à Paris rue Bodillot, $\mathrm{n}^{\circ} 90 \ldots$ pour un billet de deux cent soixante livres

[Suit la transcription sommaire de l'inventaire, fait conjointement avec Clairet ${ }^{15}$, des biens contenus dans l'appartement, grenier, cave, cuisine, chambre, et cabinet de travail ; l'estimation des biens est de 1800 livres. Est alors prise la décision] de mise en vente du mobilier dont le produit servira à payer les frais de scellés et d'inventaire...

Argent : 635 livres dont 300 en assignats.

Papiers et titres :

Liste des noms de particuliers dont Le Gray gérait des affaires...

Une inscription sur le grand livre de la dette publique non viagère, portée sur le registre A vol. 21, sous le $\mathrm{n}^{0} 24729$ de la somme annuelle de quatre vingt livres au nom du deffunt Le Gray.

(12) C'était déjà son adresse durant la Révolution.

(13) Cette propriété dont il avait hérité en 1793, située en face de l'entrée de la célèbre Cour-du-Commerce, était la pièce maîtresse de ses biens immobiliers. Les loyers qu'il en percevait constituaient une grande part de ses revenus. La maison appartenait encore à ses enfants lorsqu'elle fut acquise en 1865 par la Ville de Paris pour la somme de $232000 \mathrm{~F}$, pour être démolie lors du percement du boulevard Saint-Germain.

(14) $C f$. infra. Le carton contenait 103 pièces (titres de rentes et de propriétés immobilières), mais le détail n'est pas donné.

(15) L'inventaire après décès qui dura du 13 brumaire an VI (3 novembre 1797) au 21 frimaire an VI (11 décembre 1797) est conservé dans les minutes de $M^{\mathrm{e}}$ Clairet : AN, ET LXVI 718. 
Bail sous signature privée daté du 24 juillet 1791, avec Marie Geneviève Boudin, séparée quant aux biens de Jean Hesmart, de l'appartement qu'il occupait, pour la somme de cinq cent livres de loyer pour chaque année

[Papiers]

Cotes 1 à 18, dossiers des clients de Le Gray, classé par ordre alphabétique

[liste des noms sans prénoms ni dates]

50 pièces concernant Michel Lambert Chasles (cote 19)

96 pièces concernant Nicolas Tampin et succession Petit (cote 20)

103 pièces qui sont titres et papiers concernant le citoyen Varlet (cote 21)

29 pièces succession Farcy (cote 22)

147 pièces concernant les héritiers Piat (cote 23) ${ }^{16}$

86 pièces concernant les citoyens Lecomte et Llesogard (?) et la succession Grangeret (cote 24)

167 pièces concernant les citoyens Davigny et Marquin (cote 25)

30 pièces concernant le citoyen Chateau (cote 26)

46 pièces concernant Lemoine (cote 27 )

10 pièces qui sont registres de compte (cote 28)

différentes pièces concernant les personnes cy dessus [non classées] (cote 29)

L'expédition du mariage Legray Bodson (cote 29 [double])

Inscription sur le grand livre de la dette publique $\mathrm{n}^{\circ} 24729$ de la somme annuelle de 90 livres au nom du deffunt Legray en datte du seize messidor de l'an cinq (cote 30)

Six pièces qui sont : La première, dix actions sur la tontine Lafarge ${ }^{17}$, $\mathrm{n}^{\mathrm{o}}$ 144082-144091; La seconde, la reconnaissance d'une autre action de laditte caisse $\mathrm{n}^{\mathrm{o}} 139050$, sortie au tirage de janvier 1793 , les $3-4-5^{\mathrm{e}}$ autres,

(16) La succession Piat était si embrouillée et Legray ayant de surcroît tenté une opération immobilière douteuse en rachetant au tribunal des criées un des immeubles de cette succession (qu'il avait revendu juste avant son décès, mais sans avoir eu le temps d'en régler l'achat), que sa propre succession n'était toujours pas liquidée dix ans plus tard. Les créanciers de Legray, dont Varlet, s'étaient constitués en syndic, mais il ne semble pas qu'ils parvinrent à récupérer leurs avoirs (Union des créanciers Legray, 12 \& 26 pluviôse, 8 ventôse an VI (31 janvier 1798, 14 \& 26 février 1798), AN, ET LXVI 719). La veuve de Legray, Angélique Bodson, renonça d'ailleurs très vite à l'héritage (Veuve Legray, renonciation, 17 nivôse an VI (6 janvier 1798), AN, ET LXVI 719).

(17) AN, $\mathrm{F}^{22} 876$, Le Gray, né le 22 janvier 1754, dix actions sur la tontine Lafarge, $2^{\mathrm{e}}$ société « jeunes », $\mathrm{n}^{\circ} 144082-144091 ; 1^{\text {re }}$ société « jeunes » no 139050 , sortie au tirage de janvier 1793. 
reconnaissance de trois autres actions non sorties ; la $6^{\mathrm{e}}$ l'acte de naissance de Le Gray (cote 31)

Item, six pièces, qui sont transports, opposition, certificats de résidence et autres pièces pouvant servir de renseignement sur le compte à faire entre ledit deffunt Le Gray et de la succession de Léon François Ferdinand Salignac Fenelon, décédé évêque de Lombez (cote 32) ${ }^{18}$

Item, un écrit sous signature privée en datte à Paris du 26 mars 1791, par lequel Le Gray frère du deffunt reconnait [un prêt de 115 livres] ( cote 33)

Item, Bail sous signature privée en datte du 24 juillet 1791, [pour l'appartement de la rue des deux Boules] pour neuf années pour la somme de 500 livres annuel, à compter du $1^{\mathrm{er}}$ octobre 1791. [et un double du bail et les quittances de loyer] (cote 34)

Item, 18 pièces [de facture et quittances pour des travaux que Le Gray a fait faire dans l'appartement] (cote 35)

Item, trois pièces dont la première est l'expédition d'un acte passé devant Clairet, un des notaires soussignés, le deux ventôse de l'an cinq, duement enregistré, par lequel Charlotte Françoise Gousset, veuve d'Antoine Charles Le Gray, a reconnu avoir reçu dudit deffunt Le Gray, son fils, cinq cent vingt deux livres cinq sols et deux deniers, pour les comptes exprimés audit acte : la seconde une obligation [relative audit acte]; la troisième est l'expédition d'un acte passé devant Clairet, un des notaires soussignés, le douze mars mil sept cent quatre douze contenant la déclaration des effets appartenant à ladite veuve Le Gray dans les lieux qu'elle occupait chez le deffunt Le Gray, son fils (cote 36) ${ }^{19}$

Item, Quatre pièces qui sont quittance d'arrérage d'une rente viagère de cent quarante quatre livres due par le deffunt Le Gray, à la dame Clément... la dernière en date du deux vendémiaire de l'an quatre (cote 37)

Item, Neuf coupons de l'emprunt forcé, chacun de cent sols... (cote 38)

Trois pièces qui sont déclaration et quittances de la contribution patriotique (cote 39)

Dix pièces qui sont quittances des contributions mobilières et somptuaires dudit deffunt Le Gray pour l'année 1789 et les suivantes jusque et y compris l'an quatre et les trois cinquième de l'an cinq (cote 40)

(18) Legray avait racheté pour 4000 livres les créances sur la succession de l'évêque de Lombez, Léon François Ferdinand de Salignac de Lamothe de Fénelon, que possédait l'ancien valet de chambre du prélat, Jean-Baptiste Huguenin (AN, ET LXVI 700). Legray mena plusieurs opérations de cette nature, où il espérait que le produit de la liquidation de créances rachetées à des héritiers impatients, lui laisserait un bénéfice. Ce genre de spéculation ne lui fut pas toujours profitable...

(19) AN, ET LXVI 716. 
Quatre pièces dont la première est l'expédition d'un acte passé devant Clairet le 28 mai 1793 par lequel Le Gray a rendu le solde et compte qu'il devait en qualité d'exécuteur du testament de Margueritte Françoise Loudan, veuve de Jean Prudhomme, reçu par Clairet le 26 janvier de la même année ; les autres pièces relatives [à la même succession] (cote 41)

Quatre pièces dont la première est un pouvoir donné par le comité de surveillance et révolutionnaire de la section du Museum, le six floréal de l'an deux aux citoyens Janson et Le Gray, deux de ses membres, tant pour déposer les comptes dont il y est question que pour poursuivre l'enregistrement, déposer les sommes qui se trouveront dans... : la troisième est un écrit en datte du vingt un ventôse de l'an trois par lequel Leroux, commissaire ordonateur des guerres et agent supérieur du département de Paris a déclaré que lesdits Janson et Legray leur avaient remis un récépissé de la trésorerie nationale de la somme de quarante sept mille vingt livres datté du neuf floréal... (cote 42) ${ }^{20}$

Treize pièces qui sont mémoires d'honoraires vacations et déboursés... payés par le deffunt Legray à Clairet jusqu' au quinze ventôse de l'an cinq, et mémoires d'honoraires payés à d'autres notaires (cote 43)

Trente huit pièces qui sont minutes de procès verbaux de ventes faites par ledit deffunt Legray en qualité d'huissier priseur patenté du département de la seine les 18 et 27 frimaire an sept, 22 et 25 nivose cinq, 15 pluviose cinq, 26 ventôse sept, 9 et 18 floréal, $1^{\text {er }}$ prairial et quatorze thermidor de 1 'an quatre.... (cote 44)

Cinq pièces qui sont patentes données au deffunt Le Gray pour 1791 et 1792 , la première pour exercer la profession de receveur des rentes... (cote 45)

Vingt pièces bordereaux lettres mémoires pouvant servir de renseignement (cote 46).

Jean-Jacques TOMASSO

jjtomasso@gmail.com

(20) Sur cette mission, voir Pierre CARON, Les missions du Conseil exécutif provisoire et de la Commune de Paris dans l'Est et le Nord, août-novembre 1792, Paris, 1953. 